


\title{
Bilayer Membranes with 2D-Nematic Order of the Surfactant Polar Heads
}

\author{
J.-B. Fournier \\ Laboratoire de Physico-Chimie Théorique, E. S.P.C.I., \\ 10 rue Vauquelin, F-75231 Paris Cédex 05, France \\ P. Galatola \\ Dipartimento di Fisica, Politecnico di Torino, \\ and Istituto Nazionale di Fisica della Materia, \\ C.so Duca degli Abruzzi 24, I-10129 Torino, Italy
}

Received 08 September, 1998

\begin{abstract}
In this paper we review and discuss the possibility of having a 2D-nematic ordering of the surfactant polar heads in bilayer membranes. The coupling between the nematic order parameters and the curvature can yield instabilities in the shape of the membrane, generally leading to tubules with mesoscopic radiuses. The nematic directors in the two monolayers should present coupled disclinations with peculiar characteristics. Para-nematic membranes, i.e., nematic membranes that are in the isotropic phase when flat, should behave as the nematic membranes in the curved state.
\end{abstract}

PACS: 68.10.Et, 87.22.Bt, 64.70.Md

\section{Introduction}

Simple bilayer membranes are formed by the selfassembly of amphiphilic molecules in an aqueous environment [1]. The aliphatic chains of the constituent molecules form an oily sheet that is shielded from contact with water, on both sides, by the polar heads of the molecules. At high temperatures, in their ground state, membranes are fluid, flat and usually tensionless [2]. Their elastic energy density is therefore a function of their local curvature only [3]. Self-assembled surfactant membranes can form various phases, such as the lamellar $L_{\alpha}$ phase, in which the bilayers are staked parallel to one another, the $L_{4}$ phase, in which they form closed spherical vesicles, and the $L_{3}$ "sponge" phase, in which a single membrane is multiply connected in a random fashion. In nature, lipid membranes constitute the walls surrounding living cells, and generally host a large number of inclusions, such as cholesterol or proteins [4].

At lower temperatures, membranes can acquire varying degrees of orientational and positional order.
The molecules may tilt relative to the surface normal and develop an hexatic order in which there is a quasilong-range correlation of the six-fold bond angle. Tilted bilayers in the so-called $L_{\beta}^{\prime}$ phase tend to have both tilt and hexatic order $[5,6]$. The coupling between tilt and membrane curvature can produce a shape instability yielding elongated vesicles [7], or a "ripple" phase $\left(P_{\beta^{\prime}}\right)[8,9]$. Chiral bilayers can exhibit various phases of asymmetric ripples. In principle, membrane having in-plane nematic order are possible, however their existence has never been demonstrated. We shall argue in the following that such membranes may have already been observed.

Complex membranes can also be formed by mixing different kinds of amphiphiles [10], or adding inclusion hosts, such as it naturally occurs in biology. The coupling between the density of surfactants, or inclusions, and the membrane curvature yields several types of instabilities, generating stable spherical vesicles [11] or ripples $[12,13,14]$.

Nematic effects can result from the presence of anisotropic inclusions [15]. As an example, consider an 
integral protein, i.e., an inclusion spanning the whole bilayer, with an arbitrary shape. It will in general break both the up-down symmetry of the membrane, and its rotational in-plane symmetry, defining some direction labelled by a unit vector $\mathbf{n}$. The former effect arises for instance if the inclusion has a conical shape, and the latter if the cone angle is modulated along the perimeter of the inclusion. Purely anisotropic inclusions (with a vanishing average cone angle) are sketched in Fig. 1. In order to reduce the splay distortion of the amphiphiles bounding the inclusion, a flat membrane will tend to curve. Around an isotropic conical inclusion it will curve spherically, whereas around an anisotropic inclusion it will curve as a saddle (Fig. 1). In other words, there is a coupling between the curvature tensor $\mathbf{K}$ and the inclusion orientation $\mathbf{n}$ of the form $\mathbf{n} \cdot \mathbf{K} \cdot \mathbf{n}$. It generates a spontaneous anisotropic curvature of the membrane, favoring saddle but also tubular shapes (i.e., any shape deviating from a sphere). Because of the parity in $\mathbf{n}$ of the membrane-inclusion coupling, even if the inclusions themselves exhibit an in-plane polarity, they will orient nematically in a curved membrane, the nematic director being parallel to one of the principal axes of curvature [15]. The inclusions form then a para-nematic phase induced by the membrane curvature, which acts as an external aligning field [16].

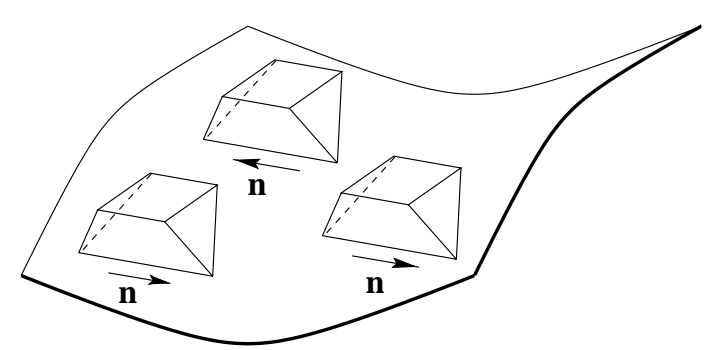

Figure 1. Purely anisotropic inclusions. They are nematically oriented by the membrane curvature they themselves produce.

In this paper we discuss the possible existence of membranes having a 2D-nematic order of their surfactant polar heads [17]. In section II we describe which kind of surfactants might yield in-plane nematic order. We discuss the stability of a flat nematic bilayer against curved states. We then show that nematic membranes should generically form tubules with a mesoscopic radius. We compare our predictions with recent experiments on membranes formed with dimeric surfactants $[18,19]$. We finally describe the peculiar defects that nematic bilayers should exhibit [20]. In section III, we discuss para-nematic membranes, i.e., membranes that are intrinsically isotropic when flat but acquire a nematic order through a curvature instability. In the curved state, para-nematic and nematic membrane should behave in the same way. Finally, we describe the various phases that nematic membranes can form.

\section{Nematic bilayers}

The usual phospholipids or surfactants forming membranes possess a double aliphatic chain and a globular polar head. In an $L_{\alpha}$ membrane, the chains form a disordered oily sheet, while the polar heads form a $2 D$ isotropic fluid on both sides. In recent years, a new class of surfactants has received increasing attention: the "gemini" [21]. They are dimeric surfactants whose polar heads are linked by a rigid spacer, which spontaneously form giant worm-like micelles [22]. Recently, another series of dimeric surfactants has been synthesized: the aggregates formed were tubular vesicles instead of the usual flat bilayers [18]. There are several independent indications that the bilayers formed have an in-plane nematic order [23]. We shall argue in the following that the formation of tubules supports this conjecture. In principle, beyond dimeric surfactants, $n$-meric surfactants with rod-like polar heads could be synthesized. In the case where their elongated heads lie in the plane of the chain-water interface, one might expect some degree of $2 \mathrm{D}$-nematic susceptibility or a true nematic order. In this section, we consider such nematic bilayers, i.e., membranes in which the polar heads of the molecules form a well-ordered 2D-nematic fluid on each side of the bilayer's oily sheet.

\section{A. Free energy density of a nematic bilayer}

The presence of an in-plane nematic ordering provides an anisotropic character to any tensorial property of the head-groups fluid, which disappears in the isotropic phase. Hence, as in bulk nematic liquid crystals [24], the order-parameter is a symmetric traceless second-rank tensor. We introduce two such orderparameters, one for each of the two head-groups fluids:

$$
\mathbf{Q}^{ \pm}=S\left(2 \mathbf{n}^{ \pm} \otimes \mathbf{n}^{ \pm}-\mathbf{I}\right)
$$

where $\mathbf{I}$ is the identity tensor, and $(\mathbf{n} \otimes \mathbf{n})_{i j}=n_{i} n_{j}$. The unit vectors $\mathbf{n}^{+}$and $\mathbf{n}^{-}$are the directors of the up- 
per and lower monolayer, which describe the directions of anisotropy associated with the two nematic orderings. $S$ is the scalar order-parameter, which describes the amount of parallel ordering of the molecules polarheads. We assume $S$ constant and identical in both monolayers.

Before discussing the free energy of a nematic bilayer, let us recall the ordinary curvature elasticity of simple membranes. At every point, the membrane's surface has two principal curvatures, $c_{1}$ and $c_{2}$, along two orthogonal axes. In the basis of the principal axes, the curvature tensor is given by

$$
\mathbf{K}=\left(\begin{array}{cc}
c_{1} & 0 \\
0 & c_{2}
\end{array}\right)
$$

The curvature energy per unit surface of a symmetric membrane, involving all quadratic terms that satisfy rotational symmetry, has the form [3]

$$
F_{0}=\frac{1}{2} \kappa\left(c_{1}+c_{2}\right)^{2}+\bar{\kappa} c_{1} c_{2}
$$

with $\kappa$ the bending rigidity and $\bar{\kappa}$ the Gaussian rigidity. One can write a similar expansion for each monolayer, however including a linear term because each of the two monolayers is individually asymmetric. Since the two monolayers have opposite curvatures, the linear terms cancel out when forming the bilayer energy (3).

Following the same procedure for a nematic membrane yields a different result. Let us write the curvature elasticity $F^{ \pm}$of each of the two nematic monolayers. Including a term linear in the curvature tensor yields the form

$$
F^{ \pm}=\frac{1}{4} \kappa\left(c_{1}+c_{2}\right)^{2}+\frac{1}{2} \bar{\kappa} c_{1} c_{2} \pm \Gamma^{ \pm}: \mathbf{K}
$$

The constants $\boldsymbol{\Gamma}^{ \pm}$are functions of the nematic orderings, i.e., at lowest order

$$
\boldsymbol{\Gamma}^{ \pm}=\alpha \mathbf{I}+\beta \mathbf{Q}^{ \pm}
$$

We have used the notation $\mathbf{\Gamma}: \mathbf{K}={ }_{i j} K_{i j}$ (summation over repeated indices being understood) and neglected the anisotropy in the elastic constants for the sake of simplicity. Summing up the two monolayers contributions yields the curvature energy density

$$
F_{\mathrm{c}}=\frac{1}{2} \kappa\left(c_{1}+c_{2}\right)^{2}+\bar{\kappa} c_{1} c_{2}+2 \beta S\left(\mathbf{n}^{+} \otimes \mathbf{n}^{+}-\mathbf{n}^{-} \otimes \mathbf{n}^{-}\right): \mathbf{K}
$$

Contrary to what occurs for simple bilayers, the linear spontaneous curvature term survives if the two directors are not parallel. Note that a mathematically similar model has been introduced for tilted membranes with a tilt-difference across the bilayer [25]. Using (2) and setting $\mathbf{n}^{ \pm}=\left(\cos \theta^{ \pm}, \sin \theta^{ \pm}\right)$yields $\left(\mathbf{n}^{ \pm} \otimes \mathbf{n}^{ \pm}\right): K=$ $\frac{1}{2}\left(c_{1}+c_{2}\right)+\frac{1}{2}\left(c_{1}-c_{2}\right) \cos 2 \theta^{ \pm}$. Therefore, the nematiccurvature coupling favors the orientation of the two di- rectors parallel to the principal axes of curvature, and mutually orthogonal in the two monolayers $\left(\theta^{+}=\pi / 2\right.$, $\theta^{-}=0$ if $\beta\left(c_{1}-c_{2}\right)>0$ and $\theta^{+}=0, \theta^{-}=\pi / 2$ if $\left.\beta\left(c_{1}-c_{2}\right)<0\right)$. This anti-nematic configuration is sketched in Fig. 2.

To the curvature energy density (6) we must add the nematic energy density

$$
F_{\mathrm{N}}=\frac{1}{2} L S^{2}\left|\nabla \mathbf{n}^{+}\right|^{2}+\frac{1}{2} L S^{2}\left|\nabla \mathbf{n}^{-}\right|^{2}-\frac{1}{2} \lambda S^{2}\left(\mathbf{n}^{+} \cdot \mathbf{n}^{-}\right)^{2}
$$

where $\left|\nabla \mathbf{n}^{ \pm}\right|^{2}=\partial_{i} n_{j}^{ \pm} \partial_{i} n_{j}^{ \pm}$. The two terms involving the elastic constant $L$ describe the elasticity associated with director distortions; the term involving the coupling constant $\lambda$ describes a weak interaction between the two nematic fields. Our model energy density for the nematic bilayer is finally $F=F_{\mathrm{c}}+F_{\mathrm{N}}$. 


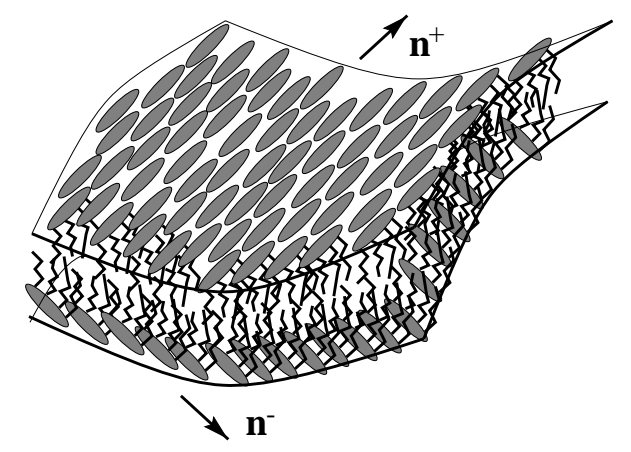

Figure 2. Nematic bilayer subject to the effect of the nematic-curvature coupling.

\section{B. Linear stability analysis}

Because of the linear curvature term in $F_{\mathrm{c}}$, the ground state of the bilayer is no longer the flat one if the two directors are non-parallel. Since this competes with the directors coupling when $\lambda>0$, a linear stability analysis is required to investigate the stability of the flat membrane.

In the vicinity of the flat state, the membrane shape can be parametrized by $z=u(x, y)$ in a rectangular coordinate frame. Calling $\theta^{ \pm}$the polar angles of the $\mathbf{n}^{ \pm}$, the membrane free energy density takes then the form

$$
\begin{aligned}
F= & \frac{1}{2} \kappa\left(u_{x x}+u_{y y}\right)^{2}+\beta S\left(u_{x x}-u_{y y}\right)\left(\cos 2 \theta^{+}-\cos 2 \theta^{-}\right)+2 \beta S u_{x y}\left(\sin 2 \theta^{+}-\sin 2 \theta^{-}\right) \\
& +\frac{1}{2} L S^{2}\left[\left(\nabla \theta^{+}\right)^{2}+\left(\nabla \theta^{-}\right)^{2}\right]+\frac{1}{2} \lambda S^{2} \sin ^{2}\left(\theta^{+}-\theta^{-}\right),
\end{aligned}
$$

where $u_{x x}=\partial^{2} u / \partial x^{2}$ and so on. We have discarded the term proportional to $\bar{\kappa}$, which is in fact a boundary term, according to the Gauss-Bonnet theorem. To study the stability of the flat state with parallel uniform directors, it is enough to develop $F$ to second order globally in $u, \theta^{+}$and $\theta^{-}$. Doing so and setting $\theta^{ \pm}=\frac{1}{2}(\phi \pm \psi)$ yields the quadratic form

$$
F \simeq \frac{1}{2} \kappa\left(u_{x x}+u_{y y}\right)^{2}+\frac{1}{4} L S^{2}(\nabla \phi)^{2}+\frac{1}{4} L S^{2}(\nabla \psi)^{2}+\frac{1}{2} \lambda S^{2} \psi^{2}+4 \beta S \psi u_{x y} .
$$

The flat membrane is unstable if there exist functions $u(x, y), \theta^{+}(x, y)$ and $\theta^{-}(x, y)$, i.e., membrane configurations, such that the latter expression is not negatively bounded. This is most easily studied in the Fourier space, in which the energy density per Fourier mode resulting from integrating (9) can be written in the following diagonalized form:

$$
\frac{1}{2} \kappa q^{4}\left|u(\mathbf{q})-\frac{4 \beta S}{\kappa} \frac{q_{x} q_{y}}{q^{4}} \psi(\mathbf{q})\right|^{2}+\frac{1}{2} S^{2}\left[\frac{1}{2} L q^{2}+\lambda-\frac{16 \beta^{2}}{\kappa} \frac{q_{x}^{2} q_{y}^{2}}{q^{4}}\right]|\psi(\mathbf{q})|^{2}+\frac{1}{4} L S^{2} q^{2}|\phi(\mathbf{q})|^{2}
$$

The flat membrane is therefore unstable for all $q$ 's such that

$$
\frac{1}{2} L q^{2}+\lambda-\frac{16 \beta^{2}}{\kappa} \frac{q_{x}^{2} q_{y}^{2}}{q^{4}}<0
$$

For a given $|\mathbf{q}|$, the most unstable mode satisfies $q_{x}=$ $q_{y}=|\mathbf{q}| / \sqrt{2}$, and then the instability condition becomes $\lambda-4 \beta^{2} / \kappa+\mathcal{O}\left(|\mathbf{q}|^{2}\right)<0$. The global instability condition is therefore

$$
\lambda<\frac{4 \beta^{2}}{\kappa} .
$$

The most unstable mode is then at $q=0$, the modula- tion occurring at $45^{\circ}$ from the direction of the initially parallel directors. The present analysis does not allow to determine the actual evolution of the membrane. If the membrane is held on a frame it might either undulate in a "ripple" or an "egg-carton" [26, 27] fashion.

Since there is usually very little interpenetration between the chains of the two monolayers forming a membrane, the coupling $\lambda$ can be estimated from anisotropic van der Waals interactions. Summing pairwise interactions yields $\lambda \simeq A_{\mathrm{a}} \ell^{2} /\left(2 \pi d^{4}\right)$, where $\ell$ is the linear size 
of the head-group, $d$ the membrane thickness, and $A_{\mathrm{a}}$ is the anisotropic Hamaker constant. Since Hamaker constants for interactions across a hydrocarbonic medium are of order $T$ [1], we take $A_{\mathrm{a}} \simeq 0.1 T$. Hence, with $d \simeq 40 \AA$ and $\ell \simeq 10 \AA$ we find $\lambda \simeq 2 \times 10^{-7} \mathrm{Jm}^{-2}$. Thus, $\lambda \ll \lambda_{\mathrm{c}}$, and therefore nematic membranes should spontaneously curve.

Freeze-fracture electron micrographs of cell membranes of Streptomyces hygroscopicus [27], which contain $40 \%$ weight fraction of cardiolipins-a diphospholipid with a double elongated polar head to which are attached four aliphatic chains-show "eggcartons" with a period in the range 200-750 . Hexagonal and 1D modulations have also been reported in similar experimental conditions. The presence of a nematic ordering of the cardiolipins might explain these unusual observations.

\section{Tubular vesicles}

In the experiment of Ref. [18], bilayers made of pure dimeric quaternary ammonium surfactants with rather long chains (typically $\mathrm{C}_{18}$ ) were shown to systematically form very long bilayer tubules. The tube radiuses were of the order of a few $1000 \AA$, since they could be seen by video-enhanced optical microscopy. Let us show that these observations are consistent with the presence of a nematic order. A cylinder is less favorable than a saddle for the nematic-curvature coupling, which is $\propto\left|c_{1}-c_{2}\right|$ once the directors are aligned along the principal axes of curvature. However it has the advantage to be compatible with uniform director alignments and it can be closed by spherical cap which avoids a contour energy.

Let us consider a closed membrane curved as a cylinder. To optimize the curvature-nematic coupling we assume $\mathbf{n}^{+}$parallel and $\mathbf{n}^{-}$perpendicular to the tube axis. Setting $c_{1}=1 / R$ and $c_{2}=0$, we obtain from (6)

$$
F_{\mathrm{tub}}=\frac{\kappa}{2} \frac{1}{R^{2}}-2 \beta S \frac{1}{R}
$$

Minimizing with respect to $R$ yields the equilibrium tube radius $R_{\mathrm{tub}}=\kappa /(2 \beta S)$.

Comparison with experiments requires evaluating $\beta$. The constants $\alpha$ and $\beta$ can be determined from the two spontaneous curvatures of a monolayer with saturated order $(S=1)$ and uniform director. Let us call $c_{\|}$(resp. $\left.c_{\perp}\right)$ the spontaneous curvature in the direction parallel (resp. perpendicular) to the director. Assuming for instance the director $\mathbf{n}^{+}$parallel to the principal axis with curvature $c_{1}$, we have

$$
F^{+}=\frac{1}{4} \kappa\left(c_{1}+c_{2}\right)^{2}+\frac{1}{2} \bar{\kappa} c_{1} c_{2}+\alpha\left(c_{1}+c_{2}\right)+\beta\left(c_{1}-c_{2}\right) \text {. }
$$

Minimizing this expression with respect to $c_{1}$ (i.e., $c_{\|}$) and $c_{2}$ (i.e., $c_{\perp}$ ) yields

$$
\begin{aligned}
\alpha & =\frac{2 \kappa+\bar{\kappa}}{4}\left(c_{\|}+c_{\perp}\right) \\
\beta & =-\frac{\bar{\kappa}}{4}\left(c_{\|}-c_{\perp}\right) .
\end{aligned}
$$

Therefore, the tube radius is

$$
R_{\mathrm{tub}}=\frac{2 \kappa}{\bar{\kappa}} \frac{1}{S\left(c_{\perp}-c_{\|}\right)} .
$$

Since the dimeric surfactants of Ref. [18] form membranes and not micelles, we expect their spontaneous curvature to be significantly larger than the monolayer thickness [1], say $100 \AA^{-1}$. The spontaneous curva- tures $c_{\|}$and $c_{\perp}$, which mainly result from packing constraints should not be very different, since the chains are quite long. Hence, it seems reasonable to assume, e.g., $c_{\perp} \simeq 120 \AA^{-1}$ and $c_{\|} \simeq 100 \AA^{-1}$. With $2 \kappa / \bar{\kappa}$ of order unity, and, e.g., $S \simeq 0.3$, we obtain $R_{\text {tub }} \approx 2000 \AA$, which is consistent with the experimental observations.

\section{Bidefects of nematic bilayers}

Due to the interaction of the two nematic fluids, nematic bilayers exhibit disclination defects with very peculiar features [20]. On a curved membrane the situation is extremely complex [28], therefore we shall re- 
strict our attention to planar bilayers. The latter could be produced by osmotically blowing up the tubes, or by patch-clamping techniques.

In terms of $\phi=\theta^{+}+\theta^{-}$and $\psi=\theta^{+}-\theta^{-}$, where, as previously, $\theta^{ \pm}$are the polar angles of the directors $\mathbf{n}^{ \pm}$, the nematic energy of a flat membrane, i.e., $F_{\mathrm{N}}$ as expressed in (7), takes the form

$$
F=\frac{1}{2} \int d^{2} r \frac{K}{2}(\nabla \phi)^{2}+\frac{1}{2} \int d^{2} r \frac{K}{2}\left\{(\nabla \psi)^{2}+\xi^{-2} \sin ^{2} \psi\right\}
$$

with $\xi^{2}=K /(2 \lambda)$. The nematic bilayer is thus equivalent to the superposition of two non-interacting nematics: a free nematic (described by $\phi$ ) and a nematic subject to a uniform field (described by $\psi$ ). The length $\xi$ is the analog of a magnetic coherence length.

The physics of defects in both the free and the fieldnematic is well known. In the latter, defects emit walls of thickness $\approx \xi$ crossing which the director turns by $\pm \pi$. From the above decomposition, we deduce that the textures generated by a defect of strength $p$ present only on the upper monolayer are given by

$$
\theta^{ \pm}=\frac{1}{2}\left(\theta_{0}(p) \pm \theta_{\lambda}(p)\right),
$$

where $\theta_{0}(p)$ describes the texture of a defect of strength $p$ in a free nematic and $\theta_{\lambda}(p)$ that in a nematic under field. Fig. 3(a) shows the director textures in the real space for $p=\frac{1}{2}$. The full (resp. dashed) lines are the field lines of the upper (resp. lower) monolayer; the dotted line indicate a wall boundary. The textures of the two monolayers coincide outside the wall. Fig. 3(b) shows the corresponding textures in the space of the free and field-nematics. The bold lines are the level lines of the free nematic, and the thin ones those of the field nematic.

More generally, one must consider pairs of associated defects in the two monolayer, i.e, bi-defects. A $[p, q]$ bi-defect is the physical superposition in the bilayer of a defect with a strength $p$ in the upper monolayer and a defect with a strength $q$ in the lower one; it is equivalent to a pair of defects of strength $p+q$ in the free nematic and of strength $p-q$ in the nematic under field. Bi-defects produce walls that reach the boundary of the sample when $p \neq q$ : the textures of the two monolayers are identical outside the walls and different in their interior. The number of wall emitted by a $[p, q]$ bi-defect is $N=2|p-q|$. The wall thickness can be determined from the value $\lambda \simeq 2 \times 10^{-7} \mathrm{Jm}^{-2}$ estimated previously. Taking, e.g., $K \simeq 3 T$ in a nematically ordered phase, we obtain $\xi=K^{1 / 2} /(2 \lambda)^{1 / 2} \simeq 1500 \AA$. The wall thickness, which is actually $\simeq 5 \xi$, should be in the $\mu \mathrm{m}$ range and thus directly observable by optical methods.

a)

b)

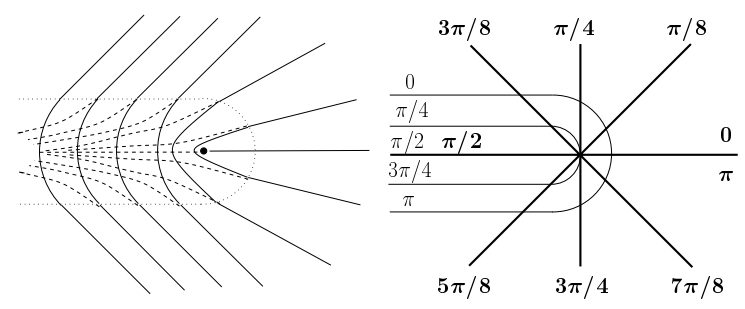

Figure 3. (a) Field lines of a $\left[\frac{1}{2}, 0\right]$ bi-defect. The full (resp. dashed) lines are the field lines of the upper (resp. lower) monolayer; the dotted line indicates the wall boundary. (b) Level lines in the free (thin lines) and field-nematics (bold lines) spaces.

\section{Para-nematic membranes}

Let us now consider the case in which the anisotropic interactions between the surfactant heads yield a large nematic susceptibility, but not a true nematic phase. This situation is reminiscent of a liquid crystal's isotropic phase in the vicinity of a nematic transition. Applying a strong external field yields a para-nematic phase with a large order-parameter [16]. Here, the situation is particularly interesting since the "external" field is actually the membrane curvature, which is an internal parameter controlled by the global energy of the system. 
Neglecting for simplicity the coupling $\mathbf{Q}^{+}: \mathbf{Q}^{-}$between the tensorial order parameters of the two monolayers and all gradient terms, the nematic energy per unit surface can be written as

$$
F_{\mathrm{N}}=\frac{1}{2} A\left(\left|\mathbf{Q}^{+}\right|^{2}+\left|\mathbf{Q}^{-}\right|^{2}\right)+B\left(\mathbf{Q}^{+}-\mathbf{Q}^{-}\right): \mathbf{K},
$$

where $\left|\mathbf{Q}^{ \pm}\right|^{2}=Q_{i j}^{ \pm} Q_{i j}^{ \pm}$. We assume $A>0$, in order for the minimum energy to correspond to the isotropic phase; the sign of $B$ is arbitrary.

Minimizing (20) with respect to the nematic tensors yields $\mathbf{Q}^{ \pm}=\mp(B / A) \tilde{\mathbf{K}}$, with $\tilde{\mathbf{K}}$ the traceless part of the curvature tensor, which is proportional to $c_{1}-c_{2}$. In the absence of thermal fluctuations, the nematic directors of the upper and lower monolayers therefore again align along the principal axes of curvature, and are perpendicular to one another.

For the following, it will be convenient to define the mean nematic ordering $\mathbf{Q}$ and the "staggered" nematic ordering $\overline{\mathbf{Q}}$, according to

$$
\mathbf{Q}=\frac{1}{2}\left(\mathbf{Q}^{(+)}+\mathbf{Q}^{(-)}\right)
$$

$$
\overline{\mathbf{Q}}=\frac{1}{2}\left(\mathbf{Q}^{(+)}-\mathbf{Q}^{(-)}\right)
$$

The free-energy $\mathcal{F}$ of the fluctuating membrane is obtained from the partition function

$$
\mathrm{e}^{-\beta \mathcal{F}}=\int \mathcal{D} \mathbf{R} \int \mathcal{D} \mathbf{Q} \int \mathcal{D} \overline{\mathbf{Q}} \mathrm{e}^{-\beta\left(F_{0}+F_{\mathrm{N}}\right)},
$$

where the integration runs over all the configurations $\mathbf{R}$ of the membrane and of the nematic order-parameter fields $\mathbf{Q}$ and $\overline{\mathbf{Q}}$. Here the parameter $\beta$ is the inverse temperature. The functional integral over $\overline{\mathbf{Q}}$ can be easily evaluated in the principal curvature frame, in which the curvature tensor (2) is diagonal. The tensor $\overline{\mathbf{Q}}$, being symmetric and traceless, can be represented in general as

$$
\overline{\mathbf{Q}}=\left(\begin{array}{cc}
\mu & \lambda \\
\lambda & -\mu
\end{array}\right)
$$

The functional integral over $\overline{\mathbf{Q}}$ is therefore proportional to

$$
\begin{aligned}
\mathrm{e}^{-\beta \mathcal{F}_{\bar{Q}}} & =\exp \left\{-\beta\left[\frac{1}{2} \kappa\left(c_{1}+c_{2}\right)^{2}+\bar{\kappa} c_{1} c_{2}\right]\right\} \int_{-\infty}^{+\infty} d \mu \exp \left\{-2 \beta\left[A \mu^{2}+B\left(c_{1}-c_{2}\right) \mu\right]\right\} \\
& =\sqrt{\frac{\pi}{2 \beta A}} \exp \left\{-\beta\left[\frac{1}{2}\left(\kappa-\frac{B^{2}}{A}\right)\left(c_{1}+c_{2}\right)^{2}+\left(\bar{\kappa}+\frac{2 B^{2}}{A}\right) c_{1} c_{2}\right]\right\} .
\end{aligned}
$$

Hence, the coupling between the nematic degrees of freedom and the curvature yields the following renormalizations of the membrane's elastic constants:

$$
\delta \kappa=-\frac{B^{2}}{A}, \quad \delta \bar{\kappa}=\frac{2 B^{2}}{A} .
$$

Taking into account the coupling between the two nematic order parameters merely adds a constant to $A$, while the gradient terms have no effects on the effective elastic constants [29]. The reduction of $\kappa$ produces curvature instabilities when $\kappa<0$. This occurs for $A \rightarrow 0$, i.e., in the vicinity of the nematic transition. The renormalization of the Gaussian modulus can drive $\bar{\kappa}$ positive, which favors the formation of saddles.

Alike renormalizations were found for isolated large anisotropic inclusions [15]. The latter tend to orient along the directions of principal curvature of the membrane, if the curvature is large enough to overcome the orientational disorder due to thermal fluctuations. If one extrapolates this result to small nematogens the size of phospholipids, one finds that the curvature required to get significant elastic constant renormalizations is of the order of the inverse membrane thickness, hence too strong for any practical situation. However, the present study shows that small nematogens can nevertheless yield strong elastic constant renormalizations provided that they already have a tendency to align parallel, i.e., possess a nematic susceptibility. The excluded volume interaction between rod-like objects and the anisotropic van der Waals forces in a dense collection of anisotropic phospholipids might yield a coeffi- 
cient $A$ small enough to provide strong elastic effects. An estimate of the nematic susceptibility coefficient $A$ has been given in the framework of a simple Onsager model [29].

\section{A. Membranes with free topology}

To study the paranematic membrane's instabilities it is necessary to introduce fourth-order terms in (20), to avoid divergences [29]. For the sake of simplicity, we make a mean-field analysis and we neglect all gradient terms. The latter approximation amounts to assuming that the membrane curvature varies slowly in space. The free-energy density generalizing (20) and including fourth-order terms is [29]

$$
F_{\mathrm{N}}^{(4)}=A|\overline{\mathbf{Q}}|^{2}+2 B \overline{\mathbf{Q}}: \mathbf{K}+\frac{1}{4} E_{1}|\overline{\mathbf{Q}}|^{4}+\frac{1}{4} E_{2} \bar{Q}_{i j} \bar{Q}_{j k} \bar{Q}_{k \ell} \bar{Q}_{\ell i}
$$

Since $A$ is assumed to be positive (the flat membrane is in its isotropic state), the mean value of $\mathbf{Q}$ is zero, not being coupled to the membrane curvature. Hence we disregarded $\mathbf{Q}$ in $F_{\mathrm{N}}^{(4)}$. Similarly, also third-order terms identically vanish and can therefore be neglected. Using (2) and (24), minimizing $F_{\mathrm{N}}^{(4)}$ with respect to $\lambda$ and $\mu$, and adding the membrane bending energy (3), gives, to fourth-order accuracy, the effective membrane's energy density

$$
F_{\mathrm{eff}}=\frac{1}{2}\left(\kappa-\frac{B^{2}}{A}\right)\left(c_{1}+c_{2}\right)^{2}+\left(\bar{\kappa}+\frac{2 B^{2}}{A}\right) c_{1} c_{2}+\frac{E B^{4}}{16 A^{4}}\left(c_{1}-c_{2}\right)^{4}
$$

where $E=E_{1}+\frac{1}{2} E_{2}>0$ for stability reasons. We recover the elastic constant renormalizations, as previously, plus a fourth-order term of nematic origin. In principle one should also consider all the fourth-order terms corresponding to the bare membrane. These are expected to be of the order of $\kappa a^{2}$, where $a$ is a microscopic length, comparable with the membrane's thickness. They are therefore negligible compared to the above fourth-order nematic contribution, provided that the nematic susceptibility $1 / A$ is large enough, which we assumed throughout. Setting

$$
c^{+}=\frac{1}{2}\left(c_{1}+c_{2}\right), \quad c^{-}=\frac{1}{2}\left(c_{1}-c_{2}\right),
$$

the effective free-energy density (28) becomes

$$
F_{\text {eff }}=(2 \kappa+\bar{\kappa}) c^{+^{2}}-\left(\bar{\kappa}+\frac{2 B^{2}}{A}\right) c^{-2}+\frac{E B^{4}}{A^{4}} c^{-4} .
$$

This shows that, since we assumed $2 \kappa+\bar{\kappa}>0$ (the bare membrane is stable in its flat state), the free-energy (30) favors a non zero $c^{-}$, i.e., structures with $c_{1} \neq c_{2}$. We shall therefore consider the possibility of sponge phases $\left(L_{3}\right)$, which correspond to a random minimal surface where everywhere the membrane is shaped as a saddle, and of tubules, where one of the two principal curvatures is zero.
To model the $L_{3}$ phase, we assume a periodic minimal structure with $c^{+}=0$ everywhere, and a characteristic cell size $h$. In average over the membrane, we then have $\left\langle c^{+^{2}}\right\rangle=0,\left\langle c^{-2}\right\rangle=M / h^{2}$ and $\left\langle c^{-4}\right\rangle=M^{\prime} / h^{4}$, where $M$ and $M^{\prime}$ are two constants that depend on the exact structure of the sponge phase. Minimizing (30) with respect to $h$, yields, when $2 B^{2} / A>-\bar{\kappa}$,

$$
h_{L_{3}}^{2}=\frac{M^{\prime}}{M} \frac{2 E B^{4}}{A^{3}} \frac{1}{A \bar{\kappa}+2 B^{2}},
$$

and the average free-energy per unit surface

$$
\langle F\rangle_{L_{3}}=-\frac{M^{2}}{M^{\prime}} \frac{A^{2}}{4 E B^{4}}\left(A \bar{\kappa}+2 B^{2}\right)^{2}
$$

This energy is negative: the $L_{3}$ phase is more stable than the flat membrane when $2 B^{2} / A>-\bar{\kappa}$. Note that $M^{2} / M^{\prime}<1$ since the $L_{3}$ phase is less optimized energetically than a uniform saddle ( $c^{-}$constant), for which we would have $M^{2}=M^{\prime}$.

In the case of tubules with radius $\mathrm{R}$, we have $c^{+}=$ $c^{-}=1 /(2 R)$. Minimizing (30) with respect to $R$ then yields, when $B^{2} / A>\kappa$,

$$
R_{\mathrm{tub}}^{2}=\frac{E B^{4}}{4 A^{3}} \frac{1}{B^{2}-A \kappa}
$$

and the free-energy density

$$
\langle F\rangle_{\mathrm{tub}}=-\frac{A^{2}}{E B^{4}}\left(B^{2}-A \kappa\right)^{2} .
$$


Therefore, the tubule state is more stable than the flat membrane for $B^{2} / A>\kappa$. The stability of the tubules relative to the $L_{3}$ phase is given by the condition [30]

$$
2 \kappa+\bar{\kappa}<2\left(\frac{\sqrt{M^{\prime}}}{M}-1\right)\left(\frac{B^{2}}{A}-\kappa\right),
$$

which defines a first-order transition line. These results are summarized in the phase diagram of Fig. 4. A general phase diagram of fourth-order membrane elastic theories for systems having internal degrees of freedom, of which the one here discussed can be considered a particular case, has been presented in Ref. [31].

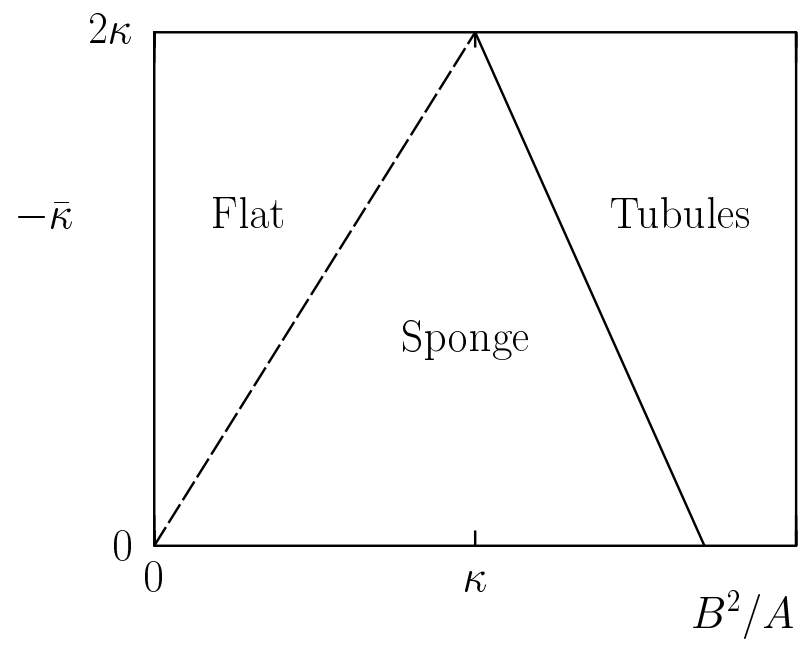

Figure 4. Phase diagram, in the space $\left(-\bar{\kappa}, B^{2} / A\right)$ of paranematic membranes with free-topology. Full (resp. dashed) lines indicate first-order (resp. second-order) transitions.

\section{B. Membranes with flat topology}

Membranes attached to a frame or spread over a hole cannot form such phases. They may however undulate in order to create a large number of local saddles ("egg-carton"). Assuming a first-order nematic transition and 1D modulations to simplify, the membrane structure can be determined by including sixth-order terms in $F_{\mathrm{N}}$ and solving numerically for the orderparameter profile [29]. The corresponding phase diagram is shown in Fig. 5. Increasing $A$ or approaching the nematic transition drives the modulation instability. The membrane is either anti-nematic (AN) or paraanti-nematic ( $\mathrm{pAN})$, according to the value of the order parameter. In the modulated $\mathrm{pAN} / \mathrm{AN}$ phase, there are regions of high and low order parameter values separated by line boundaries. The modulation wavevector is mesoscopic when the nematic transition is weakly first order, and diverges at the second-order transition.

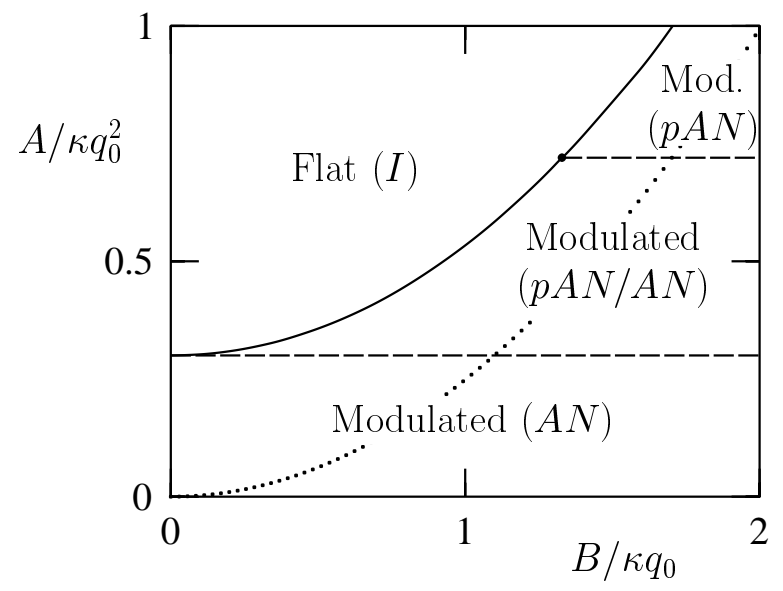

Figure 5. Phase diagram of para-nematic membranes with flat-topology. $q_{0}^{-1}$ is a length comparable to the bilayer thickness. Full (resp. dashed) lines indicate first-order (resp. second-order) transitions. Dotted line: virtual second-order transition.

\section{Conclusions}

In this paper we have analyzed the possible existence of bilayer membranes possessing a 2D-nematic order of their polar heads. Such a nematic order can arise in the presence of inclusions, as, e.g., anisotropic integral proteins or $n$-meric surfactants with polar heads. Such membranes may already have been realized [18, 19]. The coupling between the nematic order-parameter and the curvature can destabilize a flat membrane, favoring at the same time the orientation of the directors of the two monolayers parallel to the principal axes of curvature, and mutually orthogonal in the two monolayers. We have shown that nematic membranes should generically form tubules with a mesoscopic radius. Such tubules have been observed in the case of dimeric surfactants, with a radius compatible with our theoretical predictions [18]. Due to the interaction between the nematic directors of the two monolayer, nematic membranes should present disclination lines having very peculiar features [20]. To simplify, we have considered the case of flat membranes, that could be obtained by patch-clamping techniques or osmotically blowingup the tubules. We have shown that the nematic bilayer can be described in terms of two effective noninteracting nematics, a free nematic, and a nematic subject to a uniform aligning field. As a consequence, the 
presence of a defect on only one of the two monolayers gives rise to a non-trivial texture on the defect-free monolayer. We have called such defects bi-defects. Bidefects produce walls that can reach the boundary of the sample and recombine. The thickness of the walls is predicted to be in the $\mu \mathrm{m}$ range, accessible to observations by optical means.

When the anisotropic interactions between the surfactant heads are not sufficient to give rise to a nematic phase in a plane membrane, a para-nematic ordering can nevertheless develop, because of the coupling between the curvature and the nematic order-parameters, provided that the nematic susceptibility is sufficiently large. In this case, the curvature plays a role similar to that of an external aligning field in a 3D nematic in the isotropic phase, that can induce a para-nematic or a true nematic phase [16]. In the curved state, para-nematic and nematic membrane should behave in the same way. In particular, we have discussed the phase diagrams of para-nematic membranes with free and flat topology, by means of a mean field approach, taking into account higher-order terms in the nematic energy-density. In the free topology tubules and sponge phases can develop, while a membrane bound to a flat frame may undulate in an "egg-carton" fashion, in order to create a large number of local saddles, with paraanti-nematic and/or anti-nematic regions, possibly separated by line boundaries.

\section{References}

[1] J. N. Israelachvili, Intermolecular and Surface Forces (Academic Press, London, 1992).

[2] Structure and Dynamics of Membranes: From Cells to Vesicles; Generic and Specific Interactions, Vol. 1AB of Handbook of Biological Physics, edited by R. Lipowsky and E. Sackmann (North-Holland, Amsterdam, 1995).

[3] W. Helfrich, Z. Naturforsch. 28c, 693 (1973); P. Canham, J. Theor. Biol. 26, 61 (1970).

[4] H. Lodish et al., Molecular Cell Biology (Scientific American Books, New York, 1995).

[5] G. S. Smith, E. B. Sirota, C. R. Safinya, and N. A. Clark, Phys. Rev. Lett. 60, 813 (1988).

[6] G. S. Smith, E. B. Sirota, C. R. Safinya, R. J. Plano, and N. A. Clark, J. Chem. Phys. 92, 4519 (1990).
[7] F. C. MacKintosh and T. C. Lubensky, Phys. Rev. Lett. 67, 1169 (1991).

[8] A. Tardieu, V. Luzzati, and F. C. Reman, J. Mol. Biol. 75, 711 (1973)

[9] T. C. Lubensky and F. C. MacKintosh, Phys. Rev. Lett. 71, 1565 (1993).

[10] P. Hervé, D. Roux, A.-M. Bellocq, F. Nallet, and T. Gulik-Krzywicki, J. Phys. (France) II 3, 1255 (1993).

[11] E. W. Kaler, A. K. Murthy, B. E. Rodriguez, and J. A. N. Zasadzinsky, Science 245, 1371 (1989).

[12] S. Leibler, J. Phys. (Paris) 47, 507 (1986).

[13] S. A. Safran, P. A. Pincus, D. Andelman, and F. C. MacKintosh, Phys. Rev. A 43, 1071 (1991).

[14] F. C. MacKintosh and S. A. Safran, Phys. Rev. E 47, 1180 (1993).

[15] J.-B. Fournier, Phys. Rev. Lett. 76, 4436 (1996).

[16] I. Lelidis and G. Durand, Phys. Rev. E 48, 3822 (1993).

[17] A real $2 D$ nematic phase was observed for rods on the surface of water, see M. R. Fisch and C. Rosenblatt, J. Phys. (France) II 4, 103 (1994).

[18] R. Oda, I. Huc, and S. J. Candau, Chem. Commun., 2105 (1997).

[19] R. Oda, L. Bourdieu, and M. Schmutz, J. Phys. Chem. B 101, 5913 (1997).

[20] J.-B. Fournier and L. Peliti, to be published in Phys. Rev. E Rapid. Comm.

[21] R. Zana, M. Benrraou, and R. Rueff, Langmuir 7, 1072 (1991); F. M. Menger and C. A. Littau, J. Am. Chem. Soc. 113, 1451 (1991).

[22] F. Kern, F. Lequeux, R. Zana, and S. J. Candau, Langmuir 10, 1714 (1994); M. Frindi, B. Michels, H. Levy and R. Zana, Langmuir 10, 1140 (1994).

[23] S. J. Candau, private communication.

[24] P. G. de Gennes and J. Prost, The Physics of Liquid Crystals (Academic, New-York, 1993).

[25] U. Seifert, J. Shillcock, and P. Nelson, Phys. Rev. Lett. 77, 5237 (1996).

[26] R. Goetz and W. Helfrich, J. Phys. (France) II 6, 215 (1996).

[27] H. W. Meyer, W. Richter, and J. Gumper, Biochim. Biophys. Acta 1026, 171 (1990).

[28] T. C. Lubensky and J. Prost, J. Phys. (France) II 2, 371 (1992).

[29] J.-B. Fournier and P. Galatola, J. Phys. (France) II 7, 1509 (1997).

[30] Note that equation (3.12) of Ref. [29] has a misprint: its right-hand side must have a change of sign.

[31] J.-B. Fournier and P. Galatola, Europhys. Lett. 39, 225 (1997). 